\title{
Fixed point theorems for some generalized nonexpansive mappings in Ptolemy spaces
}

\section{SJ Hosseini Ghoncheh* and A Razani}

\section{"Correspondence:}

sjhghoncheh@srbiau.ac.ir

Department of Mathematics,

College of Science, Tehran Science

and Research Branch, Islamic Azad

University, Tehran, Iran

\begin{abstract}
In this paper, some existence fixed point theorems for some classes of mappings such as SCC, SKC, KSC, CSC and the class of fundamentally nonexpanisve mappings are obtained in Ptolemy spaces.

MSC: $47 \mathrm{H} 10$

Keywords: CAT(0) spaces; fundamentally nonexpansive mappings; fixed point; condition C; Ptolemy space
\end{abstract}

\section{Introduction}

Let $(X, d)$ be a metric space; the inequality

$$
d(x, y) d(z, p) \leq d(x, z) d(y, p)+d(x, p) d(y, z)
$$

is called the Ptolemy inequality, where $x, y, z, p \in X$. A Ptolemy metric space is a metric space where the Ptolemy inequality holds. It was shown in [1] that a normed space is an inner product space if and only if it is a Ptolemy space.

\section{Remark 1.1 [2]}

(I) CAT(0) spaces are Ptolemy spaces.

(II) A geodesic Ptolemy space is not necessarily a CAT(0) space (see [3] for more details).

For more details on nonexpansive mappings and related topics in fixed point theory see [4-7]. Espinola and Nicolae [8] study some properties of uniformly convex geodesic spaces in geodesic Ptolemy spaces. They prove that a geodesic Ptolemy space with a uniformly continuous midpoint map is reflexive. As a result, every bounded sequence has a unique asymptotic center. This result is used to prove some fixed point theorems for geodesic Ptolemy spaces with a uniformly continuous midpoint map. Karapınar and Tas [9] introduce some generalization of condition $C$ such as conditions $S C C, S K C, K S C$, and $C S C$, which are strong enough to generate a fixed point for discontinuous mappings. In this paper, the existence of fixed point for some class of mappings such as $S C C, S K C, K S C$, CSC, and the class of fundamentally nonexpansive mapping in Ptolemy spaces is studied.

\section{Preliminaries}

In this section, we introduce some notations (see $[8,10]$, for more details).

(2014 Hosseini Ghoncheh and Razani; licensee Springer. This is an Open Access article distributed under the terms of the Creative Commons Attribution License (http://creativecommons.org/licenses/by/2.0), which permits unrestricted use, distribution, and reproduction in any medium, provided the original work is properly cited. 
Let $(X, d)$ be a metric space; a geodesic path joining $x \in X$ to $y \in X$ is a map $c$ from a closed interval $[0, l] \subset \mathbb{R}$ to $X$ such that $c(0)=x, c(l)=y$ and $d(c(t), c(\hat{t}))=|t-\hat{t}|$ for all $t, \dot{t} \in[0, l]$. In particular, $c$ is an isometry and $d(x, y)=l$. The image $\alpha$ of $c$ is called a geodesic (or metric) segment joining $x$ and $y$. When it is unique, this geodesic is denoted by $[x, y]$. The space $(X, d)$ is said to be a geodesic space if every two points of $X$ are joined by a geodesic, and $X$ is said to be uniquely geodesic if there is exactly one geodesic joining $x$ to $y$ for each $x, y \in X$.

Definition 2.1 A subset $Y$ of a geodesic space $(X, d)$ is called convex if the geodesic segment joining any two points of $Y$ is entirely contained in $Y$.

Definition 2.2 [8] Let $X$ be a geodesic space. We say that $X$ admits a continuous midpoint map if there exists a map $m: X \times X \rightarrow X$ such that

$$
d(x, m(x, y))=d(y, m(x, y))=\frac{d(x, y)}{2} \text { for all } x, y \in X,
$$

and for $x, y, x_{n}, y_{n} \in X$, such that $\lim _{n \rightarrow \infty} d\left(x_{n}, x\right)=0$ and $\lim _{n \rightarrow \infty} d\left(y_{n}, y\right)=0$, we have $\lim _{n \rightarrow \infty} d\left(m\left(x_{n}, y_{n}\right), m(x, y)\right)=0$.

A geodesic triangle $\Delta\left(x_{1}, x_{2}, x_{3}\right)$ consist of three points $x_{1}, x_{2}$ and $x_{3}$ in $X$ (the vertex of the triangle) and three geodesic segment corresponding to each pair of points (the edges of the triangle). For the geodesic triangle $\Delta=\Delta\left(x_{1}, x_{2}, x_{3}\right)$, a comparison triangle is a triangle $\bar{\Delta}=\Delta\left(\bar{x}_{1}, \bar{x}_{2}, \bar{x}_{3}\right)$ in the Euclidean plan $\mathbb{E}^{2}$ such that $d\left(x_{i}, x_{j}\right)=d_{\mathbb{E}^{2}}\left(\bar{x}_{i}, \bar{x}_{j}\right)$ for $i, j \in\{1,2,3\}$.

A geodesic triangle $\Delta$ satisfies the CAT $(0)$ inequality if for every comparison triangle $\bar{\Delta}$ of $\Delta$ and for every $x, y \in \Delta$ we have

$$
d(x, y) \leq d_{\mathbb{E}^{2}}(\bar{x}, \bar{y})
$$

where $\bar{x}, \bar{y} \in \bar{\Delta}$ are the comparison triangle points of $x$ and $y$.

A geodesic space is $\operatorname{CAT}(0)$ space if every geodesic triangle satisfies the $\operatorname{CAT}(0)$ inequality.

Let $X$ be a metric space and $\left\{x_{n}\right\}$ be a bounded sequence in $X$. For $x \in X$ let

$$
r\left(x,\left\{x_{n}\right\}\right)=\limsup _{n \rightarrow \infty} d\left(x, x_{n}\right)
$$

The asymptotic radius $r\left(\left\{x_{n}\right\}\right)$ of $\left\{x_{n}\right\}$ in $K$ is given by

$$
r\left(K,\left\{x_{n}\right\}\right)=\inf _{x \in K} r\left(x,\left\{x_{n}\right\}\right)
$$

and the asymptotic center $A\left(\left\{x_{n}\right\}\right)$ of $\left\{x_{n}\right\}$ in $K$ is the set:

$$
A\left(K,\left\{x_{n}\right\}\right)=\left\{x \in K: r\left(x,\left\{x_{n}\right\}\right)\right\}=r\left(K,\left\{x_{n}\right\}\right) .
$$

Nonexpansive mappings and its generalization are important form applications of view. Here, we present some new generalization of this kind of mapping in Ptolemy spaces. But at first, we recall the definition of nonexpansive and quasi-nonexpansive mappings as follows. 
Definition 2.3 A map $T$ on a subset $C$ of a metric space $E$ is called nonexpansive if

$$
d(T x, T y) \leq d(x, y)
$$

for all $x, y \in C$.

Definition 2.4 A map $T$ on a subset $C$ of a metric space $E$ is called quasi-nonexpansive if

$$
d(T x, y) \leq d(x, y)
$$

for all $x \in C$ and $y \in F(T)$, where $F(T)$ is the set of all fixed points of $T$.

$$
\text { In 2008, Suzuki [11] introduced condition } C \text { as follows. }
$$

Definition 2.5 Let $T$ be a mapping on a subset $C$ of a metric space $E$. Then $T$ is said to satisfy condition $C$ if

$$
\frac{1}{2} d(x, T x) \leq d(x, y) \quad \text { implies } \quad d(T x, T y) \leq d(x, y),
$$

for all $x, y \in C$.

It is obvious that every nonexpansive mapping satisfies condition $C$, but the converse is not true. The next simple example can show this fact.

Example 2.6 [11] Define a mapping $T$ on $[0,3]$ by

$$
T(x)= \begin{cases}0 & \text { if } x \neq 3 \\ 1 & \text { if } x=3\end{cases}
$$

Then $T$ satisfies condition $C$, but $T$ is not nonexpansive.

Here, we introduce the following definition and recall some other conditions which generalize the Suzuki one:

Definition 2.7 Let $X$ be a metric space and $K$ be a subset of $X$. A mapping $T: K \rightarrow K$ is said to be fundamentally nonexpansive if

$$
d\left(T^{2} x, T y\right) \leq d(T x, y)
$$

for all $x, y \in K$.

Proposition 2.8 Every mapping which satisfies condition $C$ is fundamentally nonexpansive, but the converse is not true.

Proof By taking $x^{\prime}=T x$ and $y^{\prime}=y$, we see that every nonexpansive mapping is fundamentally nonexpansive. So by [11, Lemma 3.4 part (iii)] the result is obtained. 
Example 2.9 Suppose $X=\{(0,0),(0,1),(1,1),(1,2)\}$. Define

$$
d\left(\left(x_{1}, y_{1}\right),\left(x_{2}, y_{2}\right)\right)=\max \left\{\left|x_{1}-x_{2}\right|,\left|y_{1}-y_{2}\right|\right\}
$$

Define $T$ on $X$ by

$$
T(0,0)=(1,2), \quad T(0,1)=(0,0), \quad T(1,1)=(1,1), \quad T(1,2)=(0,1) .
$$

$T$ does not satisfy condition $C$, i.e.

$$
\frac{1}{2} d(T x, x) \leq d(x, y) \quad \text { and } \quad d(T x, T y)>d(x, y) .
$$

Let $x=(0,0), y=(0,1)$, then

$$
\frac{1}{2} d(T(0,0),(0,1)) \leq d((0,0),(0,1))
$$

and

$$
2=d(T(0,0), T(0,1))>d((0,0),(0,1))=1 .
$$

Thus condition $C$ does not hold. Now, $T$ is fundamentally nonexpansive. To show this, we show $(2.1)$ holds. Let $x=(0,0)$ and $y=(1,1)$, then

$$
d\left(T^{2}(0,0), T(1,1)\right)=d((0,1),(1,1)) \leq d(T(0,0),(1,1))=d((1,2),(1,1)) .
$$

Thus (2.1) is satisfied. Also, (2.1) holds for the other points. Moreover, in this example $T^{2}$ is not the zero constant function.

Example 2.10 Define a mapping $T$ on $[0,1]$ by

$$
T(x)= \begin{cases}0 & \text { if } x \neq 1 \\ 1 & \text { if } x=1\end{cases}
$$

It is easy to show $T$ is quasi-nonexpansive but it is not fundamentally nonexpansive mapping.

Karapınar and Tas [9] state some new definitions which are modifications of Suzuki's condition $C$, as follows.

Definition 2.11 Let $T$ be a mapping on a subset $K$ of a metric space $E$.

(i) $T$ is said to satisfy condition $S C C$ if

$$
\frac{1}{2} d(x, T x) \leq d(x, y) \quad \text { implies } \quad d(T x, T y) \leq M(x, y) \quad \text { for all } x, y \in K,
$$

where

$$
M(x, y)=\max \{d(x, y), d(x, T x), d(T y, y), d(T x, y), d(x, T y)\} .
$$


(ii) $T$ is said to satisfy condition $S K C$ if

$$
\frac{1}{2} d(x, T x) \leq d(x, y) \quad \text { implies } \quad d(T x, T y) \leq N(x, y) \quad \text { for all } x, y \in K
$$

where

$$
N(x, y)=\max \left\{d(x, y), \frac{1}{2}\{d(x, T x)+d(T y, y)\}, \frac{1}{2}\{d(T x, y)+d(x, T y)\}\right\} .
$$

(iii) $T$ is said to satisfy condition $K S C$ if

$$
\frac{1}{2} d(x, T x) \leq d(x, y) \quad \text { implies } \quad d(T x, T y) \leq \frac{1}{2}\{d(x, T x)+d(T y, y)\} \quad \text { for all } x, y \in K .
$$

(iv) $T$ is said to satisfy condition CSC if

$$
\frac{1}{2} d(x, T x) \leq d(x, y) \quad \text { implies } \quad d(T x, T y) \leq \frac{1}{2}\{d(T x, y)+d(x, T y)\} \quad \text { for all } x, y \in K .
$$

It is clear, every nonexpansive mapping satisfies condition $S K C$ ([9, Proposition 9]).

Example 2.12 Define a mapping $T$ on $[0,3]$ with the usual metric by

$$
T(x)= \begin{cases}0 & \text { if } x \neq 3 \\ 2 & \text { if } x=3\end{cases}
$$

It is obvious, $F(T)=\{0\} \neq \emptyset$ and $T$ is quasi-nonexpansive. However, since

$$
\frac{1}{2} d(3, T 3)=\frac{1}{2}|3-T 3|=\frac{1}{2} \leq 1=|3-2|=d(3,2)
$$

and

$$
d(T 3, T 2)=|T 3-T 2|=2>1=|3-2|=d(3,2),
$$

$T$ does not satisfy condition $C$, but $T$ satisfies condition $S C C$. Consider the following cases:

(1) If $x, y \in[0,3)$ then $d(T x, T y)=0 \leq M(x, y)$.

(2) If $x=y=3$ then $d(T x, T y)=d(2,2)=0 \leq M(x, y)$.

(3) If $x=3, y \in[0,3)$ then

$$
\begin{aligned}
d(T 3, T y) & =|T 3-T y|=2 \leq \max \{d(3, y), d(3, T 3), d(y, T y), d(T 3, y), d(3, T y)\} \\
& =\max \{|3-y|,|3-T 3|,|y-T y|,|T 3-y|,|3-T y|\} \\
& =\max \{|3-y|, 1,|2-y|, y, 3\} \\
& =3=M(x, y) .
\end{aligned}
$$

Karapınar and Tas [9] proved the following useful propositions. 
Theorem 2.13 Let $T$ be a mapping on a closed subset $K$ of a metric space E. Suppose $T$ satisfies condition $S K C$, then $F(T)$ is closed. Moreover, if $E$ is strictly convex and $K$ is convex, then $F(T)$ is convex.

Remark 2.14 Theorem 2.13 holds if one replaces condition $S K C$ by one of the conditions $K S C, S C C$, and $C S C$.

Theorem 2.15 Let $T$ be a mapping on a closed subset $K$ of a metric space $E$ and $T$ satisfy condition SKC, then $d(x, T y) \leq 5 d(T x, x)+d(x, y)$ holds for $x, y \in K$.

Remark 2.16 Theorem 2.15 holds if one replaces condition $S K C$ by one of the conditions $K S C$, SCC, and CSC.

See [12] for the next definition.

Definition 2.17 Let $T$ be a mapping on a subset $K$ of a metric space $X$ and $\mu \geq 1$. T is said to satisfy condition $E_{\mu}$ if

$$
d(x, T y) \leq \mu d(x, T x)+d(x, y), \quad x, y \in K .
$$

Moreover, $T$ is said to satisfy condition $E$, whenever $T$ satisfies the condition $E_{\mu}$ for some $\mu \geq 1$.

Therefore, if $T$ satisfies one of the conditions $S K C, K S C, S C C$, and $C S C$, then $T$ satisfies condition $E_{\mu}$ for $\mu=5$.

Definition 2.18 Let $X$ be a metric space, $K \subseteq X, T: K \rightarrow X$ and $\lambda \in(0,1) . T$ is said to satisfy condition $C_{\lambda}$ if

$$
\lambda d(x, T x) \leq d(x, y) \quad \text { implies } \quad d(T x, T y) \leq d(x, y), \quad \text { for all } x, y \in K .
$$

Note that, if $0<\lambda_{1}<\lambda_{2}<1$, then the condition $C_{\lambda_{1}}$ implies the condition $C_{\lambda_{2}}$. The class of mappings satisfying the condition $C_{\lambda}$ is broader than the class of mappings satisfying the condition $C$. The next lemma and theorem play important roles to obtain fixed point in the Ptolemy spaces.

Lemma 2.19 [13] Let $\left\{z_{n}\right\}$ and $\left\{w_{n}\right\}$ be bounded sequences in $K$, where $K$ is nonempty, bounded, closed, and convex subset of a metric space $X$ and $\lambda \in(0,1)$. Suppose that $z_{n+1}=$ $\lambda w_{n}+(1-\lambda) z_{n}$ and $d\left(w_{n+1}, w_{n}\right) \leq d\left(z_{n+1}, z_{n}\right)$ for all $n \in \mathbb{N}$. Then $\lim _{\sup _{n \rightarrow \infty}} d\left(w_{n}, z_{n}\right)=0$.

Theorem 2.20 [8] Let X be a complete geodesic Ptolemy space with a uniformly continuous midpoint map, $\left\{x_{n}\right\} \subseteq X$ a bounded sequence and $K \subseteq X$ is nonempty, closed, and convex. Then $\left\{x_{n}\right\}$ has a unique asymptotic center in $K$.

The following theorem can be useful for finding fixed points for nonexpansive mappings in Ptolemy spaces [8]. 
Theorem 2.21 Let $X$ be a complete geodesic Ptolemy space with a uniformly continuous midpoint map, and $K \subseteq X$ nonempty, bounded, closed, and convex. Suppose that $T: K \rightarrow$ $K$ is a nonexpansive mapping. Then $F(T)$ is nonempty, closed, and convex.

\section{Main results}

Let $X$ be complete geodesic Ptolemy space with a uniformly continuous midpoint map and $T$ be a mapping. In this section, we apply some different conditions for $T$ and give some new results for $F(T)$.

Theorem 3.1 Let $X$ be a complete geodesic Ptolemy space with a uniformly continuous midpoint map, and suppose that $K \subseteq X$ is nonempty, bounded, closed, and convex. If $T$ : $K \rightarrow K$ satisfies condition $C$, then $F(T)$ is nonempty, closed, and convex.

Proof First, there exists an approximate fixed point sequence for $T$. To show this, define a sequence $\left\{x_{n}\right\}$ in $K$ by $x_{1} \in K$ and

$$
x_{n+1}=\alpha T x_{n}+(1-\alpha) x_{n}
$$

for $n \in \mathbb{N}$, where $\alpha$ is a real number in $[1 / 2,1)$. Then, by the assumption,

$$
\frac{1}{2} d\left(x_{n}, T x_{n}\right) \leq \alpha d\left(x_{n}, T x_{n}\right)=d\left(x_{n}, x_{n+1}\right)
$$

for $n \in \mathbb{N}$, hence

$$
d\left(T x_{n+1}, T x_{n}\right) \leq d\left(x_{n+1}, x_{n}\right) .
$$

So, by Lemma 2.19

$$
\lim _{n \rightarrow \infty} d\left(x_{n}, T x_{n}\right)=0
$$

Now, by Theorem 2.20, the asymptotic center of any bounded sequence is in $K$, particularly, the asymptotic center of approximate fixed point sequence for $T$ is in $K$. Let $A\left(\left\{x_{n}\right\}\right)=\{y\}$, we show that $y$ is a fixed point of $T$. In order to prove this, one writes

$$
d\left(x_{n}, T y\right) \leq 3 d\left(T x_{n}, x_{n}\right)+d\left(x_{n}, y\right)
$$

therefore

$$
\begin{aligned}
\limsup _{n \rightarrow \infty} d\left(x_{n}, T y\right) & \leq \limsup _{n \rightarrow \infty}\left(3 d\left(T x_{n}, x_{n}\right)+d\left(x_{n}, y\right)\right) \\
& =\limsup _{n \rightarrow \infty} d\left(x_{n}, y\right) .
\end{aligned}
$$

Uniqueness of the asymptotic center implies $T y=y$.

Theorem 3.2 Let $K$ be a nonempty closed, convex, and bounded subset of a complete geodesic Ptolemy space with a uniformly continuous midpoint map $X$. Suppose that $T$ : $K \rightarrow K$ satisfies the conditions $E$ and $C_{\lambda}$ for some $\lambda \in(0,1)$. Then $T$ has a fixed point in $K$. 
Proof Define a sequence $\left\{x_{n}\right\} \subset K$ by $x_{1} \in K$ and $x_{n+1}=\lambda T x_{n}+(1-\lambda) x_{n}$ for all $n \in \mathbb{N}$. Then we have

$$
\lambda d\left(x_{n}, T x_{n}\right)=d\left(x_{n}, x_{n+1}\right) \quad(n \in \mathbb{N}) .
$$

By the condition $C_{\lambda}$, we have

$$
d\left(T x_{n}, T x_{n+1}\right) \leq d\left(x_{n}, x_{n+1}\right) \quad(n \in \mathbb{N}) .
$$

Apply Lemma 2.19 to conclude $\lim _{n \rightarrow \infty} d\left(x_{n}, T x_{n}\right)=0$.

Let $A\left(\left\{x_{n}\right\}\right)=\{w\}$, by Theorem 2.20 , we have $w \in K$. Since $T$ satisfies the condition $E$, we have

$$
d\left(x_{n}, T w\right) \leq \mu d\left(x_{n}, T x_{n}\right)+d\left(x_{n}, w\right) \quad \text { for } n \geq 1 .
$$

Taking the limit superior on both side in the above inequality, we obtain

$$
\limsup _{n \rightarrow \infty} d\left(x_{n}, T w\right) \leq \limsup _{n \rightarrow \infty} d\left(x_{n}, w\right)
$$

Uniqueness of the asymptotic center implies $T w=w$.

Theorem 3.3 Let $X$ be a complete geodesic Ptolemy space with a uniformly continuous midpoint map. Suppose that $K \subseteq X$ is nonempty, bounded, closed, and convex. If $T: K \rightarrow K$ satisfies condition SKC and $F(T) \neq \emptyset$, then $F(T)$ is closed and convex.

Proof Assume $\left(x_{n}\right)$ is a sequence in $F(T)$ which converges to some $y \in K$. We show $y \in$ $F(T)$. In order to prove this, one can write

$$
d\left(x_{n}, T y\right) \leq 5 d\left(x_{n}, T x_{n}\right)+d\left(x_{n}, y\right)
$$

therefore

$$
\begin{aligned}
\limsup _{n \rightarrow \infty} d\left(x_{n}, T y\right) & \leq \limsup _{n \rightarrow \infty}\left(5 d\left(x_{n}, T x_{n}\right)+d\left(x_{n}, y\right)\right) \\
& =\limsup _{n \rightarrow \infty} d\left(x_{n}, y\right) .
\end{aligned}
$$

Uniqueness of asymptotic center implies $T y=y . F(T)$ is convex, let $x, z \in F(T)$, then

$$
\begin{aligned}
d(x, T y) & \leq 5 d(x, T x)+d(x, y) \\
& =d(x, y)
\end{aligned}
$$

and

$$
\begin{aligned}
d(z, T y) & \leq 5 d(z, T z)+d(z, y) \\
& =d(z, y) .
\end{aligned}
$$


For $y \in[x, z]$, we have $d(x, y)+d(y, z)=d(x, z)$

$$
\begin{aligned}
d(x, z) & \leq d(x, T y)+d(T y, z) \\
& \leq d(x, y)+d(y, z) \\
& =d(x, z) .
\end{aligned}
$$

Therefore $d(x, T y)=d(x, y)$ and $d(T y, z)=d(y, z)$, because if $d(x, T y)<d(x, y)$ or $d(T y, z)<$ $d(y, z)$, then $d(x, z)<d(x, z)$ which is contradiction, therefore $T y \in[x, z]$ and $T y=y$, which means $[x, z] \subset F(T)$.

There is an example [2, p.6] to guarantee that the Ptolemy space differs from the CAT(0) space. Thanks to this example, we construct a function $T: X \rightarrow X$, where $X$ is Ptolemy, but it is not a $\operatorname{CAT}(0)$ space.

Example 3.4 Consider the space

$$
X=\{(0,0),(0,1),(1,1),(1,2)\}
$$

with $l^{\infty}$ metric,

$$
d\left(\left(x_{1}, y_{1}\right),\left(x_{2}, y_{2}\right)\right)=\max \left\{\left|x_{1}-x_{2}\right|,\left|y_{1}-y_{2}\right|\right\}
$$

$X$ is geodesic Ptolemy space, but it is not a CAT(0) space (see [2]).

Define a mapping $T$ on $X$ by

$$
T(x, y)= \begin{cases}(1,1) & \text { if }(x, y) \neq(0,0) \\ (0,1) & \text { if }(x, y)=(0,0)\end{cases}
$$

$T$ has condition $S K C$. Suppose that $(x, y)=(0,0)$ and $(x, y)=(1,1)$

$$
\frac{1}{2} d(T(0,0),(0,0)) \leq d((0,0),(1,1))
$$

and

$$
\begin{aligned}
N((0,0),(1,1))= & \max \left\{d((0,0),(1,1)), \frac{1}{2}[d(T(0,0),(0,0))+d(T(1,1),(1,1))],\right. \\
& \left.\frac{1}{2}[d(T(0,0),(1,1))+d(T(0,0),(1,1))]\right\}=1,
\end{aligned}
$$

thus

$$
d(T(0,0), T(1,1))=1 \leq N((0,0),(1,1))=1
$$

One can check that condition $S K C$ holds for the other points of the space $X$.

Note that $F(T)=\{(1,1)\} \neq \emptyset$, and $F(T)$ is closed and convex. 
Corollary 3.5 Let X be a complete geodesic Ptolemy space with a uniformly continuous midpoint map. Suppose that $K \subseteq X$ is nonempty, bounded, closed, and convex. If $T: K \rightarrow K$ satisfies condition SCC, then $F(T)$ is closed and convex.

Remark 3.6 Corollary 3.5 holds if we replace condition $S C C$ by one of the conditions KSC and CSC.

Theorem 3.7 Let $K$ be a bounded, closed, and convex subset of a complete geodesic Ptolemy space $X$ with a uniformly continues midpoint map. Suppose $T: K \rightarrow K$ satisfies condition SKC. Suppose that $\left\{x_{n}\right\}$ is a sequence in $K$ with $\lim _{n \rightarrow \infty} d\left(x_{n}, T x_{n}\right)=0$. If $A\left(\left\{x_{n}\right\}\right)=y$, then $y$ is a fixed point of $T$.

Proof Assume that there exists some approximate fixed point sequence $\left\{x_{n}\right\}$. By Theorem 2.20, the asymptotic center of any bounded sequence is in $K$, particularly, the asymptotic center of approximate fixed point sequence for $T$ is in $K$. Let $A\left(\left\{x_{n}\right\}\right)=\{y\} . y$ is a fixed point of $T$. In order to prove this, one can write

$$
d\left(x_{n}, T y\right) \leq 5 d\left(T x_{n}, x_{n}\right)+d\left(x_{n}, y\right)
$$

therefore

$$
\begin{aligned}
\limsup _{n \rightarrow \infty} d\left(x_{n}, T y\right) & \leq \limsup _{n \rightarrow \infty}\left(5 d\left(T x_{n}, x_{n}\right)+d\left(x_{n}, y\right)\right) \\
& =\limsup _{n \rightarrow \infty} d\left(x_{n}, y\right) .
\end{aligned}
$$

Uniqueness of the asymptotic center shows $T y=y$.

Corollary 3.8 Let $K$ be a bounded, closed, and convex subset of a complete geodesic Ptolemy space $X$ with a uniformly continues midpoint map. Suppose $T: K \rightarrow K$ satisfies condition SCC. Suppose that $\left\{x_{n}\right\}$ is a sequence in $K$ with $\lim _{n \rightarrow \infty} d\left(x_{n}, T x_{n}\right)=0$. If $A\left(\left\{x_{n}\right\}\right)=y$, then $y$ is a fixed point of $T$.

Remark 3.9 Corollary 3.8 holds if we replace condition $S C C$ by one of the conditions KSC and CSC.

Corollary 3.10 Let $K$ be a bounded, closed, and convex subset of a complete geodesic Ptolemy space $X$ with a uniformly continues midpoint map. Suppose $T: K \rightarrow K$ satisfies condition $E_{\mu}$. Suppose that $\left\{x_{n}\right\}$ is a sequence in $K$ with $\lim _{n \rightarrow \infty} d\left(x_{n}, \operatorname{Tx}_{n}\right)=0$. If $A\left(\left\{x_{n}\right\}\right)=y$, then $y$ is a fixed point of $T$.

Corollary 3.11 Let $K$ be a bounded, closed, and convex subset of a complete geodesic Ptolemy space $X$ with a uniformly continues midpoint map. Suppose $T: K \rightarrow K$ satisfies condition $C_{\lambda}$. Suppose that $\left\{x_{n}\right\}$ is a sequence in $K$ with $\lim _{n \rightarrow \infty} d\left(x_{n}\right.$, Tx $\left.x_{n}\right)=0$. If $A\left(\left\{x_{n}\right\}\right)=y$, then $y$ is a fixed point of $T$.

Theorem 3.12 Let $X$ be a complete geodesic Ptolemy space with a uniformly continuous midpoint map, and $K \subseteq X$ nonempty, bounded, closed, and convex. Suppose that $T: K \rightarrow$ $K$ is fundamentally nonexpansive mapping. Then $F(T)$ is nonempty, closed, and convex. 
Proof The proof is similar to the one in Theorem 3.3.

\section{Competing interests}

The authors declare that they have no competing interests.

\section{Authors' contributions}

All authors contributed equally to the writing of this paper. All authors read and approved the final manuscript.

\section{Acknowledgements}

The authors would like to thank the referees for their valuable comments, which improved the paper.

Received: 9 November 2013 Accepted: 11 March 2014 Published: 25 Mar 2014

\section{References}

1. Schoenberg, IJ: A remark on M. M. Day's characterization of inner-product spaces and conjecture of L. M. Blumenthal. Proc. Am. Math. Soc. 3, 961-964 (1952)

2. Burckley, SM, Falk, K, Wraith, DJ: Ptolemaic spaces and CAT(0). Glasg. Math. J. 51, 301-314 (2009)

3. Foertsch, T, Lytchak, A, Schroeder, V: Nonpostive curvature and the Ptolemy inequality. Int. Math. Res. Not. 2007, 100 (2007)

4. Cho, YJ, Ćirić, LB, Wang, S-H: Strong convergence theorems for nonexpansive semigroups in CAT(0) spaces. Nonlinear Anal. 74, 6050-6059 (2011)

5. Ćirić, LB: A new class of nonexpansive type mappings and fixed points. Czechoslov. Math. J. 49, 891-899 (1999)

6. Ćirić, LB: Nonexpansive type mappings and fixed point theorems in convex metric spaces. Rend. Accad. Naz. Sci. XL Mem. Mat. Appl. (5) 19, 263-271 (1995)

7. Ćirić, LB: On some nonexpansive type mappings and fixed points. Indian J. Pure Appl. Math. 24, 145-149 (1993)

8. Espinola, R, Nicolae, A: Geodesic Ptolemy spaces and fixed point. Nonlinear Anal. 74, 27-34 (2011)

9. Karapınar, E, Tas, K: Generalized (C)-conditions and related fixed point theorems. Comput. Math. Appl. 61, 3370-3380 (2011)

10. Bridson, MR, Haefliger, A: Metric Spaces of Non-Positive Curvature. Springer, Berlin (1999)

11. Suzuki, T: Fixed point theorems and convergence theorems for some generalized nonexpansive mappings. J. Math. Anal. Appl. 340, 1088-1095 (2008)

12. Dhompongsa, S, Kaewkhao, A, Panyanak, B: On Kirk's strong convergence theorem for multivalued nonexpansive mappings on CAT(0) spaces. Nonlinear Anal. 75, 459-468 (2012)

13. Goebel, K, Kirk, WA: Iteration processes for nonexpansive mappings. In: Topological Methods in Nonlinear Functional Analysis, vol. 21, pp. 115-123. Am. Math. Soc., Providence (1983)

10.1186/1687-1812-2014-76

Cite this article as: Hosseini Ghoncheh and Razani: Fixed point theorems for some generalized nonexpansive

mappings in Ptolemy spaces. Fixed Point Theory and Applications 2014, 2014:76

\section{Submit your manuscript to a SpringerOpen ${ }^{\circ}$ journal and benefit from:}

- Convenient online submission

Rigorous peer review

- Immediate publication on acceptance

Open access: articles freely available online

- High visibility within the field

- Retaining the copyright to your article 